\title{
Endoscopic endonasal approach for intracranial chordomas
}

\author{
Shamik Chakraborty ${ }^{1} \cdot$ Amir R. Dehdashti $^{1}$
}

Received: 11 November 2016/Accepted: 15 November 2016/Published online: 1 December 2016

(C) Springer-Verlag Wien 2016

The goal of any neurosurgical procedure for tumor removal involves balancing the morbidity of the operation and necessity for total resection of the tumor. This calculation becomes especially complex in regard to chordomas - relatively slowgrowing tumors, which can have devastating effects if either untreated, or improperly resected. As tumors arising from notochordal remnants, chordomas are found at the skull-base and upper spine, and at the sacrococcygeal junction. Caudal end tumors are often best treated with en-bloc resection; however, this is often not possible for skull-base tumors. Traditionally, for intracranial or skull-base chordomas, complex craniofacial procedures with high morbidity were often needed for removal, and even so often left residuals that led towards recurrence. A real gross total resection of the vast majority of chordomas is impossible, hence the reason for recurrence.

Over the past decade, the endoscopic endonasal technique, advances in instrumentation, improvements in neuroimaging, operative guidance systems, and intraoperative imaging have revolutionized skull base surgery and the treatment of these tumors. In their article, Shimony et al. [7] describe their evolution of practice in treating skull base chordomas with 17 endoscopic endonasal and 7 craniotomy procedures in 18 patients. Keeping in line with much of the recent literature, Shimony et al. found that tumors involving the midline from the sella to the first cervical vertebra are likely best approached via an endoscopic transphenoidal approach. The limitation of this technique are more laterally extending tumors. Tumors that extend into the lateral compartment of cavernous sinus,

Amir R. Dehdashti

Adehdashti@Northwell.edu

1 Department of Neurosurgery, Northshore University Hospital, Hofstra Northwell School of Medicine, Manhasset, NY, USA beyond the lateral carotid line, or lateral to the petrous apex are especially difficult to visualize, even with angled endoscopes, and are often unresectable via a pure endonasal route and necessitated further approaches/treatment. Tumors of the lower third of the clivus were found to be difficult to fully treat with this approach in their series. They concluded a $33 \%$ gross total resection based on the surgical team's assessment, but the volumetric analyses did not confirm gross total resection in any cases. This is in line with our philosophy that a true gross total resection is rarely feasible in intracranial chordomas.

Cranial nerve deficits are common in chordoma surgery and neurovascular structures are at greater risk. Sen et al. [5] had a gross total resection rate of over $85 \%$ (18 out of 21 ) in an earlier craniotomy series, but with a complication rate approaching $25 \%$ when evaluating the cranial nerve function and cerebrospinal fluid (CSF) leak, and so on. Their newer report on 71 patients had grossly comparable results with $21 \%$ CSF leak rate [6]. Sekhar et al. [4] achieved a 59\% gross total resection for the price of $21 \%$ new cranial nerve deficit and $26 \%$ CSF leak rate in another craniotomy based series. In their study, Shimony et al. [7] achieved a 33\% gross total resection (not confirmed by volumetric analyses), and a similar complication rate, with four patients in the endoscopic group (out of 17 patients) and one in the craniotomy group(out of seven patients) showing new cranial nerve deficit. Other studies, such as our earlier paper [2] using an expanded endoscopic approach for chordomas, achieved a nearly 60\% apparent gross total resection rate; however, many of the tumors in that series were not very extensive, and when using adjuvant radiation, a tumor control rate similar to that of craniotomy was achieved. That study reported a relatively low complication except for high CSF leak rate (33\%). The management of CSF leak in endonasal surgery is gradually improving with the implementation of variation of pedicled nasoseptal flaps, and as shown in the current paper, could potentially achieve a $0 \%$ 
leak rate. Larger opening at the base of the skull for extensive/ more aggressive tumors exposes the patient to an undeniably higher risk of CSF leak and therefore a more robust reconstruction technique including the use of a temporo-parietal vascularized flap is necessary.

Gross total resection is the ideal goal for these insidious lesions but, as mentioned above, is most often not possible. Given these tumors' aggressiveness and the risk involved with a true total resection, the recurrence rate remains significant. The use of adjuvant radiation maximizes tumor control and neurological preservation [1]. Careful planning and reduction of dose delivered to adjacent critical structures are important considerations [3].

Given that these tumors arise in patients with a variety of ages and symptoms, it is important to tailor their treatments appropriately and to consider a full range of surgical corridors. Endoscopic endonasal technique is one of the mainstay approaches to deal with these difficult lesions and could potentially be less invasive compared with open craniotomies in selected cases. But it necessitates an additional comprehensive multidisciplinary care, with potential need for further safe surgical resections to achieve the best possible outcomes. Considering the neurovascular complications as the main limitations of this technique, a thorough, dedicated training in endoscopic endonasal skull base surgery and experience in an endoscopic anatomical laboratory are likely to be prerequisite before tackling these formidable lesions [8].

Finally, the patient's decision, and to what extent he or she is risk averse, should be considered. A pendulum exists between a more aggressive resection, potential stroke/cranial nerve morbidity, and decreased quality of life, on one side, and a less aggressive approach with function preservation but higher risk of recurrence, on the other side. In the future, a better knowledge of molecular biology of chordoma might elucidate new opportunities to deal with these aggressive lesions.

\section{References}

1. Choy W, Terterov S, Ung $\mathrm{N}$ et al (2016) Adjuvant stereotactic radiosurgery and radiation therapy for the treatment of intracranial chordomas. J Neurol Surg B Skull Base 77:38-46

2. Dehdashti AR, Karabatsou K, Ganna A, Witterick I, Gentili F (2008) Expanded endoscopic endonasal approach for treatment of clival chordomas: early results in 12 patients. Neurosurgery 63:299-307, discussion 307-9

3. Kano H, Lunsford LD (2013) Stereotactic radiosurgery of intracranial chordomas, chondrosarcomas, and glomus tumors. Neurosurg Clin N Am 24:553-560

4. Sekhar LN, Pranatartiharan R, Chanda A, Wright DC (2001) Chordomas and chondrosarcomas of the skull base: results and complications of surgical management. Neurosurg Focus 10:E2

5. Sen C, Triana A (2001) Cranial chordomas: results of radical excision. Neurosurg Focus 10:E3

6. Sen C, Triana AI, Berglind N, Godbold J, Shrivastava RK (2010) Clival chordomas: clinical management, results, and complications in 71 patients. J Neurosurg 113:1059-1071

7. Shimoney N, Gonen L, Shofty B, Abergel A, Fliss DM, Margalit N (2016) Surgical resection of skull base chordomas: experience in case selection for surgical approach according to anatomical compartments and review of the literature. Acta Neurochir. doi:10.1007 /s00701-016-3032-9

8. Tai BL, Wang AC, Joseph JR, Wang PI, Sullivan SE, McKean EL, Shih AJ, Rooney DM (2016) A physical simulator for endoscopic endonasal drilling techniques: technical note. J Neurosurg 124:811816 